\title{
TRANSITION WAVES FOR A CLASS OF SPACE-TIME DEPENDENT MONOSTABLE EQUATIONS*
}

\author{
LUCA ROSSI ${ }^{\dagger}$ AND LENYA RYZHIK
}

\begin{abstract}
We investigate the existence of generalized transition waves for reaction-diffusion KPP equations depending explicitly on time and space. In the case of spatially periodic diffusion and drift, and general temporal dependence of the nonlinearity, we almost completely characterize the set of admissible speeds of the waves in terms of a suitable notion of mean introduced in [15]. A lower bound for the speeds is also derived for equations with non-periodic, spatially dependent coefficients, without assuming the KPP condition.
\end{abstract}

Key words. Heterogeneous reaction-diffusion equations, generalized transition waves, exponential decay.

MSC subject classification. 35K57, 35C 07 .

\section{Introduction}

We are concerned with entire solutions of the equation

$$
\partial_{t} u-\operatorname{Tr}\left(A(x) D^{2} u\right)+q(x) \cdot D u=f(t, u), \quad x \in \mathbb{R}^{N}, t \in \mathbb{R} .
$$

Here $D$ and $D^{2}$ denote respectively the gradient and the Hessian with respect to the space variables. The nonlinearity $f$ is of the KPP (or more generally monostable) type, a typical example being $f(t, u)=\mu(t) u(1-u)$, with $\mu>0$. The study of this type of equation started with the papers of Fisher [8] and Kolmogorov, Petrovskil and Piskunov [10], who considered the homogenous equation

$$
\partial_{t} u-\partial_{x x} u=f(u), \quad x \in \mathbb{R}, t \in \mathbb{R},
$$

as a model in genetics and population dynamics. It is well known for (1.2) that traveling waves of the form $u(x, t)=\phi(x-c t)$ connecting 0 and 1 exist if and only if $c$ is larger than or equal to the critical value $c_{*}=2 \sqrt{f^{\prime}(0)}$ (see $\left.[10,1]\right)$.

When the coefficients in (1.1) are periodic, the appropriate notion is a pulsating traveling wave, introduced in [23] and investigated, among many other papers, in $[24,2]$ (space dependence) and $[17,13]$ (space-time dependence).

We are interested in global in time solutions connecting the steady states $u=0$ and $u=1$ with a general temporal dependence of $f(t, u)$. The following generalization was introduced by Matano, and by Berestycki and Hamel [3].

Definition 1.1. A generalized transition wave (in the direction $e \in S^{N-1}$ ) is a timeglobal solution $u$ of $(1.1)$ such that there exists a function $c \in L^{\infty}(\mathbb{R})$ satisfying

$$
\lim _{x \cdot e \rightarrow-\infty} u\left(x+e \int_{0}^{t} c(s) d s, t\right)=1, \quad \lim _{x \cdot e \rightarrow+\infty} u\left(x+e \int_{0}^{t} c(s) d s, t\right)=0,
$$

uniformly with respect to $x \perp e$ and $t \in \mathbb{R}$. The function $c(t)$ is called the speed of the generalized transition wave $u$, and

$$
\phi(x, t):=u\left(x+e \int_{0}^{t} c(s) d s, t\right)
$$

\footnotetext{
*Received: December 6, 2012; accepted (in revised form): July 22, 2013. Communicated by Alexander Kiselev.

†Dipartimento di Matematica, Università di Padova, via Trieste 63, 35121 Padova, Italy (lucar@math.unipd.it).

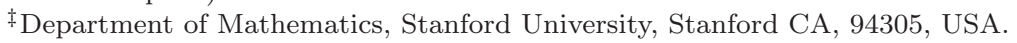


is the associated profile.

The requirement that the limits hold uniformly in $t \in \mathbb{R}$ is crucial in the above definition. The profile of a generalized transition wave satisfies

$$
\lim _{R \rightarrow-\infty} \sup _{\substack{x \cdot e<R \\ t \in \mathbb{R}}}|\phi(x, t)-1|=0, \quad \lim _{R \rightarrow+\infty} \sup _{\substack{x, e>R \\ t \in \mathbb{R}}}|\phi(x, t)|=0 .
$$

It is clear that any perturbation of $c$ obtained by adding a function with bounded integral is still a speed of $u$, though with a different profile.

To the best of our knowledge, the first examples of generalized transition waves are due to Shen [20]. Their existence in the spatially dependent non-periodic case has been proved in [18], [12] for ignition-type reaction terms. In the KPP case they do not always exist, even for large speeds, as shown in [16]. We also note that the recent paper [14] addresses the existence of critical transition waves for time-independent coefficients. In the case of KPP equations depending only on time, the range of speeds of generalized transition waves has been characterized in [21], under the assumption that the coefficients admit a uniform mean. This assumption was dropped in [15]. Finally, existence of waves with supercritical speeds is proved in [22] for equations of the form

$$
u_{t}+q(t, x) \cdot D u=\Delta u+f(t, x, u),
$$

whose coefficients are periodic in $x$ and admit uniform mean in $t$. The latter hypothesis allows the author to use the principal Lyapunov exponent and to construct transition waves with speeds admitting a uniform mean $\gamma$, for all $\gamma$ larger than some threshold.

Let us now explain what "large speed" means in the time-dependent case when the speed is neither constant in time nor expected to have averaging properties. For the space-independent KPP equation

$$
\partial_{t} u-\Delta u=f(t, u), \quad x \in \mathbb{R}^{N}, t \in \mathbb{R},
$$

Nadin and the first author were able to characterize the range of admissible speeds as follows.

Definition 1.2. The least mean (resp. the upper mean) over $\mathbb{R}$ of a function $g \in$ $L^{\infty}(\mathbb{R})$ is defined by

$$
\lfloor g\rfloor:=\lim _{T \rightarrow+\infty} \inf _{t \in \mathbb{R}} \frac{1}{T} \int_{t}^{t+T} g(s) d s, \quad\left(\text { resp. }\lceil g\rceil:=\lim _{T \rightarrow+\infty} \sup _{t \in \mathbb{R}} \frac{1}{T} \int_{t}^{t+T} g(s) d s\right) .
$$

The above notions are well posed, because they do not change if one replaces $\lim _{T \rightarrow+\infty}$ with $\sup _{T>0}\left(\right.$ resp. $\left.\inf _{T>0}\right)$ in the definition (see Proposition 3.1 in [15]). Note that $g$ admits a uniform mean $\langle g\rangle$, that is,

$$
\langle g\rangle:=\lim _{T \rightarrow+\infty} \frac{1}{T} \int_{t}^{t+T} g(s) d s
$$

exists uniformly with respect to $t \in \mathbb{R}$ if and only if $\lfloor g\rfloor=\lceil g\rceil=\langle g\rangle$. The main result of [15] is that generalized transition waves of speed $c$ with $\lfloor c\rfloor=\gamma$ exist for equation (1.5) for any $\gamma>2 \sqrt{\lfloor\mu\rfloor}$ and do not exist if $\gamma<2 \sqrt{\lfloor\mu\rfloor}$, where $\mu(t):=\partial_{u} f(t, 0)$. 
The main results. Throughout the paper, we will always assume that

$$
\begin{gathered}
\left\{\begin{array}{c}
A \text { is symmetric, uniformly continuous, } \\
\exists \alpha \geq 1, \quad \forall x \in \mathbb{R}^{N}, \quad \alpha^{-1} I \leq A(x) \leq \alpha I,
\end{array}\right. \\
q \in L^{\infty}\left(\mathbb{R}^{N}\right),
\end{gathered}
$$

and $f$ is a Carathéodory function satisfying

$$
\left\{\begin{array}{l}
\exists \delta>0, f(t, \cdot) \in W^{1, \infty}([0,1]) \cap C^{1}([0, \delta)), \text { uniformly with respect to } t \in \mathbb{R}, \\
\forall t \in \mathbb{R}, \quad f(t, 0)=f(t, 1)=0 .
\end{array}\right.
$$

We will use the notation

$$
\mu(t):=\partial_{u} f(t, 0) .
$$

Solutions of (1.1) are always understood to take values in $[0,1]$. We deal with strong solutions whose derivatives $\partial_{t}, D, D^{2}$ belong to $L^{p}\left(\mathbb{R}^{N+1}\right), \forall p<\infty$. Many of our statements are understood to hold a.e., even if we do not explicitly specify it, and inf, sup are used in place of essinf, esssup.

Our first result concerns equations with spatially periodic coefficients. Namely, we will assume that $A$ and $q$ are $l$-periodic, with $l=\left(l_{1}, \ldots, l_{N}\right) \in(0,+\infty)^{N}$, that is,

$$
\forall j \in\{1, \ldots, N\}, \forall x \in \mathbb{R}^{N}, \quad A\left(x+l_{j} e_{j}\right)=A(x), \quad q\left(x+l_{j} e_{j}\right)=q(x),
$$

where $\left(e_{1}, \ldots, e_{N}\right)$ denotes the canonical basis of $\mathbb{R}^{N}$. We will further require that

$$
\begin{gathered}
\forall t \in \mathbb{R}, u \in(0,1), \quad f(t, u) \geq 0, \quad\lfloor f(\cdot, u)\rfloor>0, \\
\forall t \in \mathbb{R}, u \in(0,1), \quad f(t, u) \leq \mu(t) u, \\
\exists C>0, \delta, \omega \in(0,1], \quad \forall t \in \mathbb{R}, u \in(0, \delta), \quad f(t, u) \geq \mu(t) u-C u^{1+\omega} .
\end{gathered}
$$

Assumption (1.10) implies that 0 and 1 are respectively unstable and stable, and (1.11) is referred to as the KPP condition. Notice that, together with (1.8), conditions (1.10) and (1.11) yield $\mu \geq 0$ and $\lfloor\mu\rfloor>0$. A sufficient condition for (1.12) to hold is $f(t, \cdot) \in C^{1+\omega}([0, \delta])$, uniformly with respect to $t$.

TheOREM 1.3. Under the assumptions (1.6)-(1.12), there exists $c_{*} \in \mathbb{R}$ such that generalized transition waves with a speed c satisfying $\lfloor c\rfloor=\gamma$ exist for all $\gamma>c_{*}$ and do not exist for $\gamma<c_{*}$.

The critical speed $c_{*}$ depends on the direction of the wave $e$ and is explicitly given by (1.16) below. Its definition involves $\lfloor\mu\rfloor$ as well as the periodic principal eigenvalues of a family of linear operators. It reduces to $2 \sqrt{\lfloor\mu\rfloor}$ for the equation (1.5). In that case, condition (2.2) below implies that the wave $u$ is decreasing in the direction $e$ and does not depend on the directions orthogonal to $e$. As we have mentioned above, the existence part of this theorem has already been proved in [22] for equation (1.4), under the additional assumption that the coefficients have uniform mean in time. However, no non-existence results are derived in [22]. 
Whereas the strategy of the proof of the existence is essentially the same as in [15], the method to prove the non-existence is new. It does not require hypotheses (1.10)-(1.12), but only $\lfloor\mu\rfloor>0$. One of the main difficulties we need to handle is that the standard preliminary step of deriving the exponential decay of the profile $\phi$ does not work, because we are not able to get estimates on $D \phi / \phi$. We overcome this difficulty by showing that, for general space time dependent problems, nonnegative entire supersolutions of linear parabolic equations which are bounded from below away from 0 in a strip cannot decay faster than a suitable exponential (see Lemma 3.1). As far as we know, this result is new, and seems to be of independent interest.

As shown in [16], transition waves for KPP nonlinearities may fail to exist even for a compact inhomogeneity. Hence, one cannot expect the existence result of Theorem 1.3 to hold without some assumptions such as the spatial periodicity. The following non-existence result holds in the general non-periodic setting.

Definition 1.4. The least mean (resp. the upper mean) over $\mathbb{R}_{-}$of a function $g \in L^{\infty}\left(\mathbb{R}_{-}\right)$is given by

$$
\left.\lfloor g\rfloor_{-}:=\lim _{T \rightarrow+\infty} \inf _{t<0} \frac{1}{T} \int_{t-T}^{t} g(s) d s, \quad \text { (resp. }\lceil g\rceil_{-}:=\lim _{T \rightarrow+\infty} \sup _{t<0} \frac{1}{T} \int_{t-T}^{t} g(s) d s\right) .
$$

THEOREM 1.5. If (1.6)-(1.8) hold and $\lfloor\mu\rfloor_{-}>0$ then there exists $c^{*} \in \mathbb{R}$ such that there are no generalized transition waves with speed $c$ satisfying $\lceil c\rceil_{-}<c^{*}$.

The formula for $c^{*}$ is given in the next section and involves a notion of generalized principal eigenvalue. If $A$ and $q$ are periodic it coincides with the one for $c_{*}$, with the only difference that the least mean of $\mu$ is taken over $\mathbb{R}_{-}$rather than $\mathbb{R}$ (which yields $\left.c^{*} \geq c_{*}\right)$. Because $\lceil c\rceil_{-}$is in general larger than $\lfloor c\rfloor$, Theorem 1.5 does not contain the non-existence result of Theorem 1.3.

Let us point out that the arguments of the proofs presented in this paper can be adapted to the case where $f$ depends on $x$ too (periodically, for the existence result) provided that $\partial_{u} f(x, t, 0)$ is of the form $\mu(t)+\tilde{\mu}(x)$.

Definition of the critical speeds $c_{*}$ and $c^{*}$. The starting point in the construction of the generalized transition waves is to find a condition under which the linearization around 0 of (1.1) admits solutions of the type $e^{-\lambda\left(x \cdot e-\int_{0}^{t} c(s) d s\right)} \varphi(x)$, with $\lambda>0$ and $\varphi$ such that $\inf _{\mathbb{R}^{N}} \varphi>0, \sup _{\mathbb{R}^{N}} \varphi<\infty$. Namely, we look for solutions of

$$
\left\{\begin{array}{l}
(\lambda c(t)-\mu(t)) \varphi=L_{\lambda} \varphi, \quad x \in \mathbb{R}^{N}, t \in \mathbb{R}, \\
\inf _{\mathbb{R}^{N}} \varphi>0, \quad \sup _{\mathbb{R}^{N}} \varphi<\infty
\end{array}\right.
$$

with

$$
L_{\lambda} \psi:=\operatorname{Tr}\left(A(x) D^{2} \psi\right)-2 \lambda e A(x) D \psi-q(x) \cdot D \psi+\left(\lambda^{2} e A(x) e+\lambda q(x) e\right) \psi .
$$

It follows that $\lambda c(t)-\mu(t)$ is constant in $t$ and it is an eigenvalue of $L_{\lambda}$, with an associated eigenfunction $\varphi_{\lambda}$ which is bounded and has positive infimum. A sufficient condition to solve this problem is that $A$ and $q$ are $l$-periodic. Indeed, in this case, there exists a unique (simple) eigenvalue $k(\lambda)$, called the principal eigenvalue, with a positive, $l$-periodic eigenfunction $\varphi_{\lambda}$, called the principal eigenfunction. It is known that $\lambda \mapsto k(\lambda)$ is convex on $\mathbb{R}$ (see the arguments in the proof of Proposition 5.7 part (iii) of [2]). Moreover, $k(0)=0$ and

$$
\forall \lambda \in \mathbb{R}, \quad \inf _{x \in \mathbb{R}^{N}}\left(\lambda^{2} e A(x) e+\lambda q(x) e\right) \leq k(\lambda) \leq \sup _{x \in \mathbb{R}^{N}}\left(\lambda^{2} e A(x) e+\lambda q(x) e\right),
$$


as seen by evaluating the expression $k(\lambda) \varphi_{\lambda}=L_{\lambda} \varphi_{\lambda}$ at a minimum and a maximum point of $\varphi_{\lambda}{ }^{1}$. These properties imply that the following definition for the critical speed $c_{*}$ is well posed if $\lfloor\mu\rfloor>0$ :

$$
c_{*}:=\min _{\lambda>0} \frac{\lfloor\mu\rfloor+k(\lambda)}{\lambda} .
$$

The non-existence result of Theorem 1.3 is also obtained by use of solutions of (1.13).

Instead, Theorem 1.5 is derived using an approach in which only subsolutions of (1.13) are involved. This is why $A$ and $q$ are not required to be periodic. The role of $k(\lambda)$ is played by the following quantity:

$$
\tilde{k}(\lambda):=\sup \left\{k \in \mathbb{R}: \exists \varphi \in W_{l o c}^{2, N}\left(\mathbb{R}^{N}\right) \cap L^{\infty}\left(\mathbb{R}^{N}\right), \inf _{\mathbb{R}^{N}} \varphi>0, L_{\lambda} \varphi \geq k \varphi \text { in } \mathbb{R}^{N}\right\} .
$$

This is a notion of generalized principal eigenvalue which has been introduced in [4] and is reminiscent of the ones considered in $[5,6]$. As we show in Appendix A, under the hypotheses $(1.6),(1.7)$, the above quantity is a well defined real number. Recalling Definition 1.4, we set

$$
c^{*}:=\min _{\lambda>0} \frac{\lfloor\mu\rfloor_{-}+\tilde{k}(\lambda)}{\lambda} .
$$

The existence of the above minimum when $\lfloor\mu\rfloor_{-}>0$ is guaranteed by Corollary A.3. If $A$ and $q$ are $l$-periodic then $\tilde{k}(\lambda)=k(\lambda)$ by Corollary A.2 (notice that the inequality $\tilde{k}(\lambda) \geq k(\lambda)$ simply follows by taking $\varphi=\varphi_{\lambda}$ in (1.17)) and thus $c^{*} \geq c_{*}$.

REMARK 1.1. Theorem 1.5 can be stated in the following slightly stronger form: let $\left(x_{n}\right)_{n}$ be such that $A\left(x+x_{n}\right) \rightarrow A^{*}(x), q\left(x+x_{n}\right) \rightarrow q^{*}(x)$ locally uniformly in $x$, and let $\tilde{k}^{*}(\lambda)$ be given by (1.17) with $A, q$ replaced by $A^{*}, q^{*}$. Then there are no generalized transition waves with speed $c$ satisfying

$$
\lceil c\rceil<\min _{\lambda>0} \frac{\lfloor\mu\rfloor+\tilde{k}^{*}(\lambda)}{\lambda} .
$$

This is seen by applying Theorem 1.5 to the limit of the translations of the generalized transition wave by (a subsequence of) $\left(x_{n}, t_{n}\right)_{n}$, where $t_{n}$ is such that $\left(x_{n} \cdot e-\int_{0}^{t_{n}} c(s) d s\right)_{n}$ is bounded.

\section{Proof of the existence part of Theorem 1.3}

Consider $\gamma>c_{*}$, with $c_{*}$ given by (1.16). We know that the function $k$ is convex ([2]) and analytic ([9]). Hence, it is strictly convex. Because it vanishes at 0 and has quadratic growth, we see that the equation $\lfloor\mu\rfloor+k(\lambda)=\gamma \lambda$ has exactly two positive solutions. Call the smallest one $\lambda$. We then define the speed $c$ in the following way:

$$
c(t):=\frac{\mu(t)+k(\lambda)}{\lambda} .
$$

It follows that $\lfloor c\rfloor=\gamma$. Moreover, $\varphi=\varphi_{\lambda}$ satisfies (1.13) and then, by (1.11), the function $w$ defined by

$$
w(x, t):=\min \left(e^{-\lambda\left(x \cdot e-\int_{0}^{t} c(s) d s\right)} \varphi_{\lambda}(x), 1\right)
$$

\footnotetext{
${ }^{1}$ Because $\varphi_{\lambda}$ is not $C^{2}$, one actually makes use of the Bony maximum principle [7] to derive, say, at a minimum point $\hat{x}, k(\lambda) \varphi_{\lambda}(\hat{x})=\lim \operatorname{essup}_{x \rightarrow \hat{x}} L_{\lambda} \varphi_{\lambda}(x) \geq \lim \operatorname{essup}_{x \rightarrow \hat{x}}(\lambda e A(x) e+q(x) e) \varphi_{\lambda}(\hat{x})$.
} 
is a generalized supersolution of (1.1).

Next, in order to construct a subsolution, take $\omega$ so that (1.12) holds. By our choice of $\lambda$, there exists $\lambda^{\prime} \in(\lambda,(1+\omega) \lambda)$ such that

$$
\frac{\lfloor\mu\rfloor+k\left(\lambda^{\prime}\right)}{\lambda^{\prime}}<\gamma=\frac{\lfloor\mu\rfloor+k(\lambda)}{\lambda} .
$$

We then set

$$
\psi(x, t):=e^{\sigma(t)-\lambda^{\prime}\left(x \cdot e-\int_{0}^{t} c(s) d s\right)} \varphi_{\lambda^{\prime}}(x),
$$

with a function $\sigma \in W^{1, \infty}(\mathbb{R})$ that will be specified later. We have

$$
\begin{aligned}
\partial_{t} \psi-\operatorname{Tr}\left(A(x) D^{2} \psi\right)+q(x) \cdot D \psi-\mu(t) \psi & =\left[\sigma^{\prime}(t)+\lambda^{\prime} c(t)-k\left(\lambda^{\prime}\right)-\mu(t)\right] \psi \\
& =\left[\sigma^{\prime}(t)+B(t)\right] \psi
\end{aligned}
$$

with

$$
B(t):=\mu(t)\left(\frac{\lambda^{\prime}}{\lambda}-1\right)+\frac{\lambda^{\prime}}{\lambda} k(\lambda)-k\left(\lambda^{\prime}\right)
$$

We now make use of the following crucial property of the least mean (see Lemma 3.2 of [15]):

$$
\forall g \in L^{\infty}(\mathbb{R}), \quad\lfloor g\rfloor=\sup _{\sigma \in W^{1, \infty}(\mathbb{R})} \inf _{t \in \mathbb{R}}\left(\sigma^{\prime}+g\right)(t) .
$$

Because

$$
\lfloor B\rfloor=\lfloor\mu\rfloor\left(\frac{\lambda^{\prime}}{\lambda}-1\right)+\frac{\lambda^{\prime}}{\lambda} k(\lambda)-k\left(\lambda^{\prime}\right)=\lambda^{\prime}\left(\frac{\lfloor\mu\rfloor+k(\lambda)}{\lambda}-\frac{\lfloor\mu\rfloor+k\left(\lambda^{\prime}\right)}{\lambda^{\prime}}\right)>0,
$$

by (2.1) we can choose $\sigma \in W^{1, \infty}(\mathbb{R})$ so that $\beta:=\inf _{\mathbb{R}}\left(\sigma^{\prime}+B\right)>0$, and, as a consequence,

$$
\partial_{t} \psi-\operatorname{Tr}\left(A(x) D^{2} \psi\right)+q(x) \cdot D \psi \geq(\mu(t)+\beta) \psi, \quad x \in \mathbb{R}^{N}, t \in \mathbb{R} .
$$

For $m>0$ large enough, the function $v$ defined by

$$
v(x, t):=\max \left(e^{-\lambda\left(x \cdot e-\int_{0}^{t} c(s) d s\right)} \varphi_{\lambda}(x)-m \psi(x, t), 0\right)
$$

vanishes for $x \cdot e-\int_{0}^{t} c(s) d s \leq 0$. If $v(x, t)>0$ (and then $x \cdot e-\int_{0}^{t} c(s) d s>0$ ), we get

$$
\begin{aligned}
& \partial_{t} v-\operatorname{Tr}\left(A(x) D^{2} v\right)+q(x) \cdot D v-\mu(t) v \leq-m \beta \psi \\
= & -m \beta\left[e^{-\lambda\left(x \cdot e-\int_{0}^{t} c(s) d s\right)} \varphi_{\lambda}(x)\right]^{1+\omega} \frac{\varphi_{\lambda^{\prime}}(x)}{\varphi_{\lambda}^{1+\omega}(x)} e^{\sigma(t)-\left(\lambda^{\prime}-(1+\omega) \lambda\right)\left(x \cdot e-\int_{0}^{t} c(s) d s\right)} \\
\leq & -m \beta v^{1+\omega} \inf _{y \in \mathbb{R}^{N}} \frac{\varphi_{\lambda^{\prime}}(y)}{\varphi_{\lambda}^{1+\omega}(y)} \inf _{s \in \mathbb{R}} e^{\sigma(s)} .
\end{aligned}
$$

We have used the fact that $\lambda^{\prime}<(1+\omega) \lambda$ in the last step. Consequently, by hypothesis (1.12), we can choose $m$ large enough so that $v$ is a generalized subsolution of (1.1) smaller than 1 . 
With a subsolution and a supersolution in hand, a solution $v \leq u \leq w$ can be obtained in a standard way, as the limit of (a subsequence of) the solutions $\left(u_{n}\right)_{n \in \mathbb{N}}$ of the problems

$$
\left\{\begin{array}{l}
\partial_{t} u_{n}-\operatorname{Tr}\left(A(x) D^{2} u_{n}\right)+q(x) \cdot D u_{n}=f\left(t, u_{n}\right), \quad x \in \mathbb{R}^{N}, t>-n, \\
u_{n}(x,-n)=w(x,-n), \quad x \in \mathbb{R}^{N}
\end{array}\right.
$$

We immediately get

$$
\lim _{x \cdot e \rightarrow+\infty} u\left(x+e \int_{0}^{t} c(s) d s, t\right) \leq \lim _{x \cdot e \rightarrow+\infty} w\left(x+e \int_{0}^{t} c(s) d s, t\right)=0,
$$

uniformly in $x \perp e, t \in \mathbb{R}$.

It remains to prove that

$$
\lim _{x \cdot e \rightarrow-\infty} u\left(x+e \int_{0}^{t} c(s) d s, t\right)=1,
$$

also uniformly in $x \perp e, t \in \mathbb{R}$. Set

$$
\vartheta:=\lim _{R \rightarrow-\infty} \inf _{\substack{x, e<R \\ t \in \mathbb{R}}} u\left(x+e \int_{0}^{t} c(s) d s, t\right) .
$$

Our goal is to show that $\vartheta=1$. Let $\left(x_{n}\right)_{n \in \mathbb{N}}$ in $\mathbb{R}^{N}$ and $\left(t_{n}\right)_{n \in \mathbb{N}}$ in $\mathbb{R}$ be such that

$$
\lim _{n \rightarrow \infty} x_{n} \cdot e=-\infty, \quad \lim _{n \rightarrow \infty} u\left(x_{n}+e \int_{0}^{t_{n}} c(s) d s, t_{n}\right)=\vartheta .
$$

For $n \in \mathbb{N}$, let $k_{n} \in \prod_{j=1}^{N} l_{j} \mathbb{Z}$ be such that $y_{n}:=x_{n}+e \int_{0}^{t_{n}} c(s) d s-k_{n} \in \prod_{j=1}^{N}\left[0, l_{j}\right)$ and define $\psi_{n}(x, t):=u\left(x+k_{n}, t+t_{n}\right)$. The functions $\left(\psi_{n}\right)_{n \in \mathbb{N}}$ are solutions of

$$
\partial_{t} \psi_{n}-\operatorname{Tr}\left(A(x) D^{2} \psi_{n}\right)+q(x) \cdot D \psi_{n}=f\left(t+t_{n}, \psi_{n}\right), \quad x \in \mathbb{R}^{N}, t \in \mathbb{R} .
$$

By parabolic estimates, we find that $\left(\psi_{n}\right)_{n \in \mathbb{N}}$ converges (up to subsequences) locally uniformly to some function $\psi$ satisfying

$$
\partial_{t} \psi-\operatorname{Tr}\left(A(x) D^{2} \psi\right)+q(x) \cdot D \psi=g(x, t), \quad x \in \mathbb{R}^{N}, t \in \mathbb{R},
$$

where $g$ is the weak limit in $L_{l o c}^{p}\left(\mathbb{R}^{N} \times \mathbb{R}\right)$ of (a subsequence of) $f\left(t+t_{n}, \psi_{n}(x, t)\right.$ ). Furthermore, letting $y$ be the limit of (a converging subsequence of) $\left(y_{n}\right)_{n \in \mathbb{N}}$, we find that $\psi(y, 0)=\vartheta$ and

$$
\forall x \in \mathbb{R}^{N}, t \in \mathbb{R}, \quad \psi(x, t)=\lim _{n \rightarrow \infty} u\left(x+x_{n}+e \int_{0}^{t_{n}} c(s) d s-y_{n}, t+t_{n}\right) \geq \vartheta .
$$

As a consequence, because $g \geq 0$, the strong maximum principle yields $\psi=\vartheta$ in $\mathbb{R}^{N} \times$ $(-\infty, 0]$. In particular, we must have $g=0$ in $\mathbb{R}^{N} \times(-\infty, 0)$. Using the Lipschitz continuity of $f(t, \cdot)$, we get

$$
\forall T>0, \quad 0=\lim _{n \rightarrow \infty} \int_{B_{1}} d x \int_{-T}^{0} f\left(t+t_{n}, \psi_{n}(x, t)\right) d t=\left|B_{1}\right| \lim _{n \rightarrow \infty} \int_{t_{n}-T}^{t_{n}} f(t, \vartheta) d t .
$$

It follows that $\lfloor f(\cdot, \vartheta)\rfloor \leq 0$, whence $\vartheta=0$ or 1 due to (1.10). We will conclude by showing that $\vartheta>0$. This inequality would be straightforward if $u$ were nonincreasing 
in the direction $e$, but this in general is not the case. However, the following property suffices:

$$
\forall x \in \mathbb{R}^{N}, t \in \mathbb{R}, h \in \prod_{j=1}^{N} l_{j} \mathbb{Z}, \quad h \cdot e(u(x, t)-u(x+h, t)) \geq 0 .
$$

This property is inherited by $u_{n}$ - and then by $u$ - from $w$, due to the maximum principle. For $R \in \mathbb{R}$, call $A_{R}:=\left\{x \in \mathbb{R}^{N}: x \cdot e<R\right\}$. Take $R \in \mathbb{R}$ and $x \in A_{R}$. Letting $j \in\{1, \ldots, N\}$ be such that $e \cdot e_{j} \neq 0$, we can find $z \in \mathbb{Z}$ such that

$$
R \leq x \cdot e+n l_{j} e_{j} \cdot e<R+|l| .
$$

Namely, $x+n l_{j} e_{j} \in A_{R+|l|} \backslash A_{R}$. Because $n l_{j} e_{j} \cdot e \geq R-x \cdot e>0$, (2.2) yields $u(x, t) \geq$ $u\left(x+n l_{j} e_{j}, t\right)$ for all $t \in \mathbb{R}$. This shows that, for $R \in \mathbb{R}$,

$$
\inf _{\substack{x \in A_{R} \\ t \in \mathbb{R}}} u(x, t) \geq \inf _{\substack{x \in A_{R+|l|} \backslash A_{R} \\ t \in \mathbb{R}}} u(x, t) .
$$

Whence, because $u \geq v$, we derive

$$
\inf _{\substack{x \in A_{R} \\ t \in \mathbb{R}}} u\left(x+e \int_{0}^{t} c(s) d s, t\right) \geq \inf _{\substack{x \in A_{R+\mid}|| \mid \backslash A_{R} \\ t \in \mathbb{R}}} v\left(x+e \int_{0}^{t} c(s) d s, t\right) .
$$

It follows from the construction of $v$ that the latter term is strictly positive for $R$ large enough. Recalling the definition of $\vartheta$, we eventually infer that $\vartheta>0$. This finishes the proof of the existence part of Theorem 1.3.

\section{The non-existence results}

3.1. Outline of the proof. A possible approach to prove the non-existence of waves with a slow speed is that of Mallordy and Roquejoffre [11], constructing subsolutions with support contained in infinite strips. This is achieved in the spatially periodic case by perturbation of the eigenvalue problems for $k(\lambda)$, which are analytic in $\lambda$. In the non-periodic case, we do not know if the generalized principal eigenvalues replacing $k(\lambda)$ are analytic and thus the above method cannot be used. We develop here a different approach which applies to both theorems 1.3 and 1.5. In particular, this provides a new proof of the result in the time independent, spatially periodic case.

The starting point-Lemma 3.1 below - is that any supersolution of a linear parabolic equation in $\mathbb{R}^{N} \times(-\infty, \tau)$, which is bounded from below away from zero for $0 \leq x \cdot e \leq 1$ and $t<\tau$, cannot decay faster than a suitable exponential in the direction $e$. This is used slightly differently in the periodic case (Thereom 1.3) and non-periodic case (Theorem 1.5). In the periodic case the "not too fast" exponential decay of transition waves allows us to build below them subsolutions that move "with high speeds", and that prohibits too slow transition waves. The construction of the subsolution requires a slow modulation of the exponential decay rate for which we need some results from the operator perturbation theory obtained in Section 3.3. This subsolution construction is realized in Section 3.4 for the proof of the non-existence result of Theorem 1.3. Theorem 1.5 (the non-periodic case) is proved in Section 3.5. There, we use the subsolution to relate the upper mean of the speed to the "optimal" exponential decay rate $\lambda_{0}$. More precisely, we show that if the the speed is too slow in terms of $\lambda_{0}$ then we may construct a subsolution with an exponential decay which is initially faster than $\lambda_{0}$ but, as time goes on, becomes slower, leading to a contradiction. 
3.2. Exponential decay of positive entire supersolutions. In order to prove the non-existence results in theorems 1.3 and 1.5, we first obtain two auxiliary results on the exponential decay of the profile of a generalized transition wave. We prove these results in the general framework of positive entire supersolutions of spacetime dependent equations.

To set the notation, for a given function $\phi: \mathbb{R}^{N} \times \mathbb{R} \rightarrow \mathbb{R}$ we define

$$
\begin{aligned}
& \Lambda_{\phi}:=\left\{\lambda>0: \inf _{\substack{x, e>0 \\
t \in \mathbb{R}}} \phi(x, t) e^{\lambda x \cdot e}>0\right\}, \\
& \Lambda_{\phi}^{-}:=\left\{\lambda>0: \inf _{\substack{x, e>0 \\
t<0}} \phi(x, t) e^{\lambda x \cdot e}>0\right\} .
\end{aligned}
$$

Roughly speaking, $\Lambda_{\phi}$ and $\Lambda_{\phi}^{-}$are the sets of exponential decays faster than $\phi$ for $t \in \mathbb{R}$ and $t<0$ respectively. We first derive a sufficient condition for those sets to be nonempty when $\phi$ is a supersolution of a parabolic equation. Then we show that they are bounded from below by a positive constant depending on the coefficients of the equation. The latter is only used in the proof of Theorem 1.5.

Lemma 3.1. Let $\phi$ be a nonnegative supersolution of

$$
\partial_{t} w-\operatorname{Tr}\left(A(x, t) D^{2} w\right)+q(x, t) \cdot D w+h(x, t) w=0, \quad x \in \mathbb{R}^{N}, t<\tau,
$$

with $\tau \in \mathbb{R} \cup\{+\infty\}, A, q, h \in L^{\infty}\left(\mathbb{R}^{N} \times(-\infty, \tau)\right)$, and $A$ uniformly elliptic. There exists then $\lambda>0$ depending on $A, q$ and $h$, such that, for $x \cdot e>0$ and $t<\tau$,

$$
\phi(x, t) \geq\left(\inf _{\substack{0 \leq x \cdot e \leq 1 \\ t<\tau}} \phi(x, t)\right) e^{-\lambda x \cdot e} .
$$

Proof. Set $\beta:=\inf _{\substack{0 \leq x \cdot e \leq 1 \\ t<\tau}} \phi(x, t)>0$. For $n \in \mathbb{N}$ satisfying $n>-\tau$, define, for $x \in \mathbb{R}^{N}$ and $-n \leq t<\tau$,

$$
\eta_{n}(x, t):= \begin{cases}\beta\left(1-\frac{x \cdot e}{t+n+1}\right)^{\lambda(t+n+1)}, & \text { if } x \cdot e<t+n+1, \\ 0, & \text { otherwise. }\end{cases}
$$

The value $\lambda>0$ will be chosen later. For $-n<t<\tau$ and $0<x \cdot e<t+n+1$, we set $\rho_{n}:=\left(1-\frac{x \cdot e}{t+n+1}\right) \in(0,1)$ and compute

$$
\begin{aligned}
& \frac{\rho_{n}^{2}}{\eta_{n}}\left(\partial_{t} \eta_{n}-\operatorname{Tr}\left(A D^{2} \eta_{n}\right)+q \cdot D \eta_{n}+h \eta_{n}\right) \\
= & \lambda \rho_{n}^{2} \ln \rho_{n}+\frac{x \cdot e}{t+n+1} \lambda \rho_{n}-e A e\left(\lambda^{2}-\frac{\lambda}{t+n+1}\right)-\lambda q \cdot e \rho_{n}+h \rho_{n}^{2} \\
\leq & \lambda\left(1-\alpha^{-1} \lambda+\alpha+\|q\|_{\infty}\right)+\|h\|_{\infty} .
\end{aligned}
$$

Here $\alpha \geq 1$ is such that $\alpha^{-1} I \leq A \leq \alpha I$. Hence, for $\lambda$ large enough, depending on $\alpha$, $q$, $h$, the function $\eta_{n}$ is a generalized subsolution of (3.1) for $x \cdot e>0,-n<t<\tau$. In addition, if $x \cdot e=0$ then $\eta_{n}(x, t)=\beta \leq \phi(x, t)$, and if $x \cdot e>0$ then $\eta_{n}(x,-n) \leq \beta_{(0,1)}(x$. $e) \leq \phi(x,-n)$. By comparison we get $\eta_{n} \leq \phi$ for $x \cdot e>0,-n<t<\tau$. Because this is true for every $n \in \mathbb{N}$, we get

$$
\phi(x, t) \geq \beta \lim _{n \rightarrow \infty}\left(1-\frac{x \cdot e}{t+n+1}\right)^{\lambda(t+n+1)}=\beta e^{-\lambda x \cdot e},
$$


for all $x \in \mathbb{R}^{N}$ such that $x \cdot e>0$, and all $t<\tau$.

We will make use of the analogue of $(2.1)$ for the least mean on $\mathbb{R}_{-}$:

$$
\forall g \in L^{\infty}\left(\mathbb{R}_{-}\right), \quad\lfloor g\rfloor_{-}=\sup _{\sigma \in W^{1, \infty}\left(\mathbb{R}_{-}\right)} \inf _{t<0}\left(\sigma^{\prime}+g\right)(t) .
$$

This formula easily follows from (2.1) by noticing that $\lfloor g\rfloor_{-}=\lfloor g(-|\cdot|)\rfloor$.

Lemma 3.2. Let $\phi$ be a nonnegative generalized supersolution of

$$
\partial_{t} w-\operatorname{Tr}\left(A(x, t) D^{2} w\right)+q(x, t) \cdot D w=f(x, t, w), \quad x \in \mathbb{R}^{N}, t<0,
$$

with $A, q \in L^{\infty}\left(\mathbb{R}^{N} \times \mathbb{R}_{-}\right)$, A uniformly elliptic, and $f(x, t, u)$ Lipschitz continuous in $u$. Assume that there exist $\delta>0$ and $g \in L^{\infty}\left(\mathbb{R}_{-}\right)$with $\lfloor g\rfloor_{-}>0$ such that

$$
\forall x \in \mathbb{R}^{N}, t<0,0<u<\delta, \quad f(x, t, u) \geq g(t) u .
$$

Suppose further that

$$
\inf _{\substack{x \cdot e>0 \\ t<0}} \phi(x, t)=0,
$$

and that $\Lambda_{\phi}^{-} \neq \emptyset$. Then $\inf \Lambda_{\phi}^{-} \geq \tilde{\lambda}>0$, where $\tilde{\lambda}$ depends on the ellipticity constants of $A,\|q\|_{L^{\infty}\left(\mathbb{R}^{N} \times \mathbb{R}_{-}\right)}$, and $\lfloor g\rfloor_{-}$.

Proof. Let $\lambda \in \Lambda_{\phi}^{-}$. There exists a positive constant $\beta$ such that

$$
\forall x \in \mathbb{R}^{N}: x \cdot e \geq 0, t<0, \quad \phi(x, t) \geq \beta e^{-\lambda x \cdot e} .
$$

By (3.2), there exists $\sigma \in W^{1, \infty}\left(\mathbb{R}_{-}\right)$such that $\left(\sigma^{\prime}+g\right)(t)>\frac{1}{2}\lfloor g\rfloor_{-}$for $t<0$. Set $\tilde{\beta}:=$ $\min \left(\beta e^{-\|\sigma\|_{\infty}-2 \lambda}, \delta e^{-\|\sigma\|_{\infty}}\right)$ and, for $n \in \mathbb{N}$, define

$$
\forall x \in \mathbb{R}^{N},-n \leq t<0, \quad \theta_{n}(x, t):=\tilde{\beta} e^{-\sigma(t)-\lambda \chi\left(\frac{x \cdot e}{t+n+1}\right) x \cdot e},
$$

with $\chi \in C^{2}(\mathbb{R})$ satisfying

$$
\chi=0 \text { in }(-\infty, 1), \quad \chi=1 \text { in }(2,+\infty), \quad \chi^{\prime} \geq 0 \text { in } \mathbb{R} .
$$

It follows that $\sup \theta_{n} \leq \delta$ and that $\theta_{n} \leq \phi$ for $x \cdot e=0,-n \leq t \leq 0$ and for $x \cdot e \geq 0, t=-n$. We claim that, for $\lambda$ small enough, $\theta_{n}$ is a subsolution of (3.3) for $t>-n$. To verify that, for $x \in \mathbb{R}^{N}, t>-n$, setting $\rho:=\frac{x \cdot e}{t+n+1}$ we find that

$$
\begin{aligned}
& \frac{1}{\theta_{n}}\left(\partial_{t} \theta_{n}-\operatorname{Tr}\left(A D^{2} \theta_{n}\right)+q \cdot D \theta_{n}\right) \\
= & -\sigma^{\prime}+\lambda \rho^{2} \chi^{\prime}(\rho)+\lambda e A e\left[\lambda\left(\chi(\rho)+\rho \chi^{\prime}(\rho)\right)^{2}-\frac{2 \chi^{\prime}(\rho)+\rho \chi^{\prime \prime}(\rho)}{t+n+1}\right]-\lambda q \cdot e\left(\chi(\rho)+\rho \chi^{\prime}(\rho)\right) .
\end{aligned}
$$

Because $\chi$ is constant outside $(1,2)$, we can find a constant $C$, depending on the ellipticity constant of $A,\|q\|_{L^{\infty}\left(\mathbb{R}^{N+1}\right)}$, and $\|\chi\|_{C^{2}(\mathbb{R})}$, such that

$$
\partial_{t} \theta_{n}-\operatorname{Tr}\left(A D^{2} \theta_{n}\right)+q \cdot D \theta_{n}<\left(g-\frac{1}{2}\lfloor g\rfloor_{-}+C \lambda(\lambda+1)\right) \theta_{n}, \quad x \in \mathbb{R}^{N}, t>-n .
$$

Assume, for the sake of contradiction, that $C \lambda(\lambda+1) \leq \frac{1}{2}\lfloor g\rfloor_{-}$. Then (3.4) implies that $\theta_{n}$ is a subsolution of (3.3) for $t>-n$. By the parabolic comparison principle we infer that $\phi \geq \theta_{n}$ for $x \cdot e \geq 0,-n<t<0$. This is true for all $n \in \mathbb{N}$, whence

$$
\forall x \in \mathbb{R}^{N}: x \cdot e>0, t<0, \quad \phi(x, t) \geq \lim _{n \rightarrow \infty} \theta_{n}(x, t)=\tilde{\beta} e^{-\|\sigma\|_{\infty}} .
$$

This contradicts (3.5). Consequently, $C \lambda(\lambda+1)>\frac{1}{2}\lfloor g\rfloor_{-}$, which finishes the proof. 
3.3. Perturbation results for the principal eigenfunction of periodic linear operators. We now derive some regularity properties of the $\phi_{\lambda}$ with respect to $\lambda$ that we will need below. In this section, $L$ denotes an operator of the form

$$
L \psi:=\operatorname{Tr}\left(A(x) D^{2} \psi\right)-q(x) \cdot D \psi .
$$

We assume that

$$
\left\{\begin{array}{l}
A \text { is symmetric, } l \text {-periodic, continuous with modulus } \omega, \\
\exists \alpha \geq 1, \quad \forall x \in \mathbb{R}^{N}, \quad \alpha^{-1} I \leq A(x) \leq \alpha I, \\
q \in L^{\infty}\left(\mathbb{R}^{N}\right) \text { is } l \text {-periodic, } \\
\exists \beta>0, \quad\|A\|_{L^{\infty}\left(\mathbb{R}^{N}\right)},\|q\|_{L^{\infty}\left(\mathbb{R}^{N}\right)} \leq \beta
\end{array}\right.
$$

Given $\lambda \in \mathbb{R}, L_{\lambda}$ denotes the operator (1.14) and $k(\lambda), \varphi_{\lambda}$ are the associated periodic principal eigenvalue and eigenfunction. In order to stress the dependence on $\lambda$, we will sometimes write $\Phi(\lambda, \cdot)$ in place of $\varphi_{\lambda}(\cdot)$. Because $L_{\lambda}$ depends analytically on $\lambda$, it follows from the classical perturbation theory that the same is true for $k$ and $\Phi$, provided that $\Phi$ is normalized by, say, $\Phi(\lambda, 0)=1$ (see $[9,19]$ ). We then set

$$
\dot{\varphi}_{\lambda}(x):=\partial_{\lambda} \Phi(\lambda, x), \quad \ddot{\varphi}_{\lambda}(x):=\partial_{\lambda}^{2} \Phi(\lambda, x) .
$$

Theorem 3.3. Assume that L satisfies (3.6). Then

$$
\forall \underline{\lambda} \leq \lambda \leq \bar{\lambda}, \quad\left\|\varphi_{\lambda}\right\|_{W^{1, \infty}\left(\mathbb{R}^{N}\right)} \leq C_{1}, \quad\left\|\dot{\varphi}_{\lambda}\right\|_{W^{1, \infty}\left(\mathbb{R}^{N}\right)} \leq C_{2}, \quad\left\|\ddot{\varphi}_{\lambda}\right\|_{W^{1, \infty}\left(\mathbb{R}^{N}\right)} \leq C_{3},
$$

where $C_{1}$ depends on $N,|l|, \omega, \alpha, \beta, \underline{\lambda}$, and $\bar{\lambda}, C_{2}$ depends on these terms as well as $k^{\prime}(\underline{\lambda})$ and $k^{\prime}(\bar{\lambda})$, and $C_{3}$ depends on these terms and $\max _{\underline{\lambda} \leq \eta \leq \bar{\lambda}} k^{\prime \prime}(\eta)$.

Proof. Notice first that, by (1.15), for $\underline{\lambda} \leq \lambda \leq \bar{\lambda},|k(\lambda)|$ is controlled by $\beta, \underline{\lambda}$, and $\bar{\lambda}$. Because $\varphi_{\lambda}$ is $l$-periodic and equal to 1 at 0 , the $W^{1, \infty}$ estimate for $\varphi_{\lambda}$ follows from the Harnack inequality and classical interior estimates.

We now derive the $L^{\infty}$ estimate for $\dot{\varphi}_{\lambda}$. Differentiating the equation $\left(L_{\lambda}-\right.$ $k(\lambda)) \Phi(\lambda, x)=0$ with respect to $\lambda$, we find that $\dot{\varphi}_{\lambda}$ is an $l$-periodic solution of

$$
\left(L_{\lambda}-k(\lambda)\right) \dot{\varphi}_{\lambda}=k^{\prime}(\lambda) \varphi_{\lambda}+2 e A(x) D \varphi_{\lambda}-(2 \lambda e A(x) e+q(x) e) \varphi_{\lambda}, \quad x \in \mathbb{R}^{N} .
$$

Moreover, because $\Phi(\lambda, 0)=1$ for all $\lambda$, it follows that $\dot{\varphi}_{\lambda}(0)=0$.

Assume for the sake of contradiction that there exists a sequence $\left(\lambda_{n}\right)_{n \in \mathbb{N}}$ in $[\underline{\lambda}, \bar{\lambda}]$ and a family of operators $\left(L^{n}\right)_{n \in \mathbb{N}}$ of the form $L^{n}=\operatorname{Tr}\left(A^{n} D^{2}\right)-q^{n} \cdot D$, satisfying (3.6) with $l, \omega, \alpha, \beta$ independent of $n$, for which $\left(k_{n}^{\prime}(\underline{\lambda})\right)_{n \in \mathbb{N}},\left(k_{n}^{\prime}(\bar{\lambda})\right)_{n \in \mathbb{N}}$ are bounded and

$$
\lim _{n \rightarrow \infty}\left\|\dot{\varphi}_{\lambda_{n}}^{n}\right\|_{L^{\infty}\left(\mathbb{R}^{N}\right)}=\infty .
$$

Here, for $n \in \mathbb{N}$ and $\lambda \in \mathbb{R}, L_{\lambda}^{n}, k_{n}(\lambda), \varphi_{\lambda}^{n}$, and $\dot{\varphi}_{\lambda}^{n}$ denote the analogues of $L_{\lambda}, k(\lambda)$, $\varphi_{\lambda}$, and $\dot{\varphi}_{\lambda}$, with $L$ replaced by $L^{n}$. We have that, as $n \rightarrow \infty$ (up to subsequences),

$$
\lambda_{n} \rightarrow \lambda \in[\underline{\lambda}, \bar{\lambda}], \quad A^{n} \rightarrow \tilde{A} \text { in } L^{\infty}\left(\mathbb{R}^{N}\right), \quad q^{n} \rightarrow \tilde{q} \text { in } L_{l o c}^{p}\left(\mathbb{R}^{N}\right), \forall p<\infty .
$$

Let $\tilde{L}_{\lambda}$ be given by (1.14) with $A, q$ replaced by $\tilde{A}, \tilde{q}$ and call $k(\lambda), \varphi_{\lambda}$ the associated periodic principal eigenvalue and eigenfunction, normalized by $\varphi_{\lambda}(0)=1$. Using the boundedness of $\left(k_{n}\left(\lambda_{n}\right)\right)_{n \in \mathbb{N}}$ and the uniform estimates on the $\varphi_{\lambda_{n}}^{n}$ one readily sees that, as $n \rightarrow \infty$,

$$
k_{n}\left(\lambda_{n}\right) \rightarrow k(\lambda), \quad \varphi_{\lambda_{n}}^{n} \rightarrow \varphi_{\lambda} \text { in } W^{1, \infty}\left(\mathbb{R}^{N}\right)
$$


Consider now $\left(x_{n}\right)_{n \in \mathbb{N}}$ such that

$$
\forall n \in \mathbb{N}, \quad\left|\dot{\varphi}_{\lambda_{n}}^{n}\left(x_{n}\right)\right|=\left\|\dot{\varphi}_{\lambda_{n}}^{n}\right\|_{L^{\infty}\left(\mathbb{R}^{N}\right)} .
$$

By periodicity, we may assume that $x_{n}$ converges (up to subsequences) to some $\tilde{x}$ as $n \rightarrow \infty$. Up to changing sign to $\dot{\varphi}_{\lambda_{n}}^{n}$ if $\dot{\varphi}_{\lambda_{n}}^{n}\left(x_{n}\right)<0$, we have that the $\dot{\varphi}_{\lambda_{n}}^{n}$ satisfy

$$
\dot{\varphi}_{\lambda_{n}}^{n}\left(x_{n}\right)=\left\|\dot{\varphi}_{\lambda_{n}}^{n}\right\|_{L^{\infty}\left(\mathbb{R}^{N}\right)}, \quad\left(L_{\lambda_{n}}^{n}-k_{n}\left(\lambda_{n}\right)\right) \dot{\varphi}_{\lambda_{n}}^{n}=g_{n}(x), \quad x \in \mathbb{R}^{N},
$$

where $\left\|g_{n}\right\|_{L^{\infty}\left(\mathbb{R}^{N}\right)}$ is controlled by $N, \beta, \underline{\lambda}, \bar{\lambda},\left\|\varphi_{\lambda_{n}}^{n}\right\|_{W^{1, \infty}\left(\mathbb{R}^{N}\right)}$, and $k_{n}^{\prime}\left(\lambda_{n}\right)$. The latter is bounded, because the convexity of $k_{n}$ yields $k_{n}^{\prime}(\underline{\lambda}) \leq k_{n}^{\prime}\left(\lambda_{n}\right) \leq k_{n}^{\prime}(\bar{\lambda})$. Hence, $\left(\left\|g_{n}\right\|_{L^{\infty}\left(\mathbb{R}^{N}\right)}\right)_{n \in \mathbb{N}}$ is bounded. The functions $\left(\psi_{n}\right)_{n \in \mathbb{N}}$ defined by

$$
\psi_{n}(x):=\frac{\dot{\varphi}_{\lambda_{n}}^{n}(x)}{\left\|\dot{\varphi}_{\lambda_{n}}^{n}\right\|_{L^{\infty}\left(\mathbb{R}^{N}\right)}}
$$

satisfy, for $n \in \mathbb{N}$,

$$
\psi_{n}(0)=0, \quad \psi_{n}\left(x_{n}\right)=\left\|\psi_{n}\right\|_{L^{\infty}\left(\mathbb{R}^{N}\right)}=1, \quad\left(L_{\lambda_{n}}^{n}-k_{n}\left(\lambda_{n}\right)\right) \psi_{n}=\frac{g_{n}}{\left\|\dot{\varphi}_{\lambda_{n}}^{n}\right\|_{L^{\infty}\left(\mathbb{R}^{N}\right)}} .
$$

Thus, the interior estimates imply that (up to subsequences) $\left(\psi_{n}\right)_{n \in \mathbb{N}}$ converges to a periodic function $\psi$ satisfying

$$
\psi(0)=0, \quad \psi(\tilde{x})=\|\psi\|_{L^{\infty}\left(\mathbb{R}^{N}\right)}=1, \quad\left(\tilde{L}_{\lambda}-k(\lambda)\right) \psi=0, \quad x \in \mathbb{R}^{N} .
$$

The simplicity of $k(\lambda)$ then implies that $\psi$ coincides with $\varphi_{\lambda}$ up to a scalar multiple. This is impossible because $\psi(0)=0$ and $\psi(\tilde{x})=1$.

We have proved the $L^{\infty}$ bound for $\dot{\varphi}_{\lambda}$. Because $\dot{\varphi}_{\lambda}$ satisfies $(3.7)$, the $W^{1, \infty}$ bound follows from a priori estimates. Finally, in order to get the result for $\ddot{\varphi}_{\lambda}$, one differentiates the equation (3.7) with respect to $\lambda$ and argues as before (notice that $\ddot{\varphi}_{\lambda}$ vanishes at 0 too).

REMARK 3.1. The above arguments apply to arbitrary analytic families of linear elliptic operators. In such cases, bootstrapping gives

$$
\forall n \in \mathbb{N}, \underline{\lambda} \leq \lambda \leq \bar{\lambda}, \quad\left\|\partial_{\lambda}^{n} \Phi(\lambda, \cdot)\right\|_{W^{1, \infty}\left(\mathbb{R}^{N}\right)} \leq C\left(N,|l|, \omega, \alpha, \beta, \underline{\lambda}, \bar{\lambda}, n, \max _{\substack{j=1, \ldots, n \\ \underline{\lambda \leq n \leq \lambda}}} k^{(j)}(\eta)\right) .
$$

3.4. The spatially periodic case. We first construct a bounded subsolution $\underline{v}$ of the linear equation

$$
\partial_{t} u-\operatorname{Tr}\left(A(x) D^{2} u\right)+q(x) \cdot D u-g(t) u=0, \quad x \in \mathbb{R}^{N}, t>0,
$$

with a prescribed exponential decay $\lambda_{0}$, whose level lines approximately move with speed

$$
\min _{0<\lambda \leq \lambda_{0}} \frac{\lfloor g\rfloor_{+}+k(\lambda)}{\lambda} .
$$

Here, $\lfloor g\rfloor_{+}$denotes the least mean of $g$ on $\mathbb{R}_{+}$:

$$
\lfloor g\rfloor_{+}:=\lim _{T \rightarrow+\infty} \inf _{t>0} \frac{1}{T} \int_{t}^{t+T} g(s) d s .
$$


The basic idea is to connect the function $e^{\lambda_{0}(x \cdot e-\gamma t)} \varphi_{\lambda_{0}}(x)$ to $\varphi_{0}(x)$ as $x \cdot e-\gamma t \rightarrow-\infty$ by slowly decreasing $\lambda_{0}$ and using the estimates of Theorem 3.3. Here, for $\lambda \geq 0, \varphi_{\lambda}$ is the periodic principal eigenfunction of $L_{\lambda}$ normalized by $\varphi_{\lambda}(0)=1$. As in Section 3.3 , we use the notation

$$
\Phi(\lambda, x):=\varphi_{\lambda}(x), \quad \dot{\varphi}_{\lambda}(x):=\partial_{\lambda} \Phi(\lambda, x), \quad \ddot{\varphi}_{\lambda}(x):=\partial_{\lambda}^{2} \Phi(\lambda, x) .
$$

We further need a more precise version of (2.1), provided by Remark 3.3 in [15], which estimates the $L^{\infty}$ norm of $\sigma$ : for $g \in L^{\infty}\left(\mathbb{R}_{+}\right)$and $T>0$, there exists $\sigma \in W^{1, \infty}\left(\mathbb{R}_{+}\right)$ such that

$$
\inf _{\mathbb{R}_{+}}\left(\sigma^{\prime}+g\right)=\inf _{t>0} \frac{1}{T} \int_{t}^{t+T} g(s) d s, \quad\|\sigma\|_{L^{\infty}\left(\mathbb{R}_{+}\right)} \leq 2 T\|g\|_{L^{\infty}\left(\mathbb{R}_{+}\right)} .
$$

Lemma 3.4. Assume that $A$, q satisfy (1.6), (1.7), and (1.9) and let $g \in L^{\infty}\left(\mathbb{R}_{+}\right)$, $\lambda_{0}>0, \gamma \in \mathbb{R}$ be such that

$$
\lfloor g\rfloor_{+}>0, \quad \gamma<\min _{0<\lambda \leq \lambda_{0}} \frac{\lfloor g\rfloor_{+}+k(\lambda)}{\lambda} .
$$

Then, there exist $T, \varepsilon>0$ such that

$$
\inf _{t>0} \frac{1}{T} \int_{t}^{t+T} g(s) d s \geq \max _{0 \leq \lambda \leq \lambda_{0}}(\gamma \lambda-k(\lambda))+\varepsilon,
$$

and a positive subsolution $\underline{v}$ of (3.8) satisfying

$$
\kappa \leq \underline{v}(x, t) \leq 1 \quad \text { if } x \cdot e \leq \gamma t, \quad \underline{v}(x, t) \leq e^{-\lambda_{0}(x \cdot e-\gamma t)} \quad \text { if } x \cdot e>\gamma t,
$$

where $\kappa \in(0,1)$ depends on $T, \varepsilon, N,|l|, \alpha, \lambda_{0}, \gamma, k^{\prime}(0), k^{\prime}\left(\lambda_{0}\right), \max _{0 \leq \eta \leq \lambda_{0}} k^{\prime \prime}(\eta)$, the modulus of continuity of $A$, and the $L^{\infty}\left(\mathbb{R}^{N}\right)$ norms of $A, q$, and $\mu$. because

Proof. The existence of $T, \varepsilon>0$ satisfying (3.10) follows from the hypotheses,

$$
\max _{0 \leq \lambda \leq \lambda_{0}}(\gamma \lambda-k(\lambda))<\lfloor g\rfloor_{+} .
$$

We refer for brevity to the terms $\kappa$ is claimed to depend on in the statement of the lemma as the "desired terms". Let $\chi$ be a nonincreasing smooth function satisfying

$$
\chi=1 \text { in }(-\infty, 0], \quad \chi=0 \text { in }[1,+\infty), \quad\left\|\chi^{\prime}\right\|_{L^{\infty}(\mathbb{R})}+\left\|\chi^{\prime \prime}\right\|_{L^{\infty}(\mathbb{R})} \leq H,
$$

where $H$ is a universal constant. Set

$$
\eta(z):= \begin{cases}\lambda_{0} \chi(\tilde{\varepsilon} \ln (1-z)), & \text { if } z<0 \\ \lambda_{0}, & \text { if } z \geq 0,\end{cases}
$$

where $0<\tilde{\varepsilon} \leq 1$ will be chosen later. Then define

$$
v(x, t):=e^{-(x \cdot e-\gamma t) \eta(x \cdot e-\gamma t)} \Phi(\eta(x \cdot e-\gamma t), x) .
$$

Calling $P$ the linear operator in $(3.8)$ and $w(z):=e^{-z \eta(z)}$, direct computation yields

$$
\begin{aligned}
P v=- & \gamma\left(w^{\prime} \varphi_{\eta}+w \eta^{\prime} \dot{\varphi}_{\eta}\right)-w \operatorname{Tr}\left(A D^{2} \varphi_{\eta}\right)-2 w^{\prime} e A D \varphi_{\eta}-w^{\prime \prime} e A e \varphi_{\eta} \\
& -2 \eta^{\prime} e A\left(e w^{\prime} \dot{\varphi}_{\eta}+w D \dot{\varphi}_{\eta}\right)-w e A e\left(\eta^{\prime \prime} \dot{\varphi}_{\eta}+\left(\eta^{\prime}\right)^{2} \ddot{\varphi}_{\eta}\right) \\
& +q \cdot\left[e w^{\prime} \varphi_{\eta}+w\left(D \varphi_{\eta}+e \eta^{\prime} \dot{\varphi}_{\eta}\right)\right]-g(t) w \varphi_{\eta}
\end{aligned}
$$


where the argument of $w, \eta$ (and their derivatives) is $x \cdot e-\gamma t$, while that of $\varphi_{\lambda}, \dot{\varphi}_{\lambda}$, $\ddot{\varphi}_{\lambda}$ is $x$. Because $\eta$ satisfies

$$
\forall z \in \mathbb{R}, \quad\left|\eta^{\prime}(z)\right| \leq \frac{\tilde{\varepsilon} \lambda_{0} H}{1+|z|}, \quad\left|\eta^{\prime \prime}(z)\right| \leq \frac{\tilde{\varepsilon} \lambda_{0} H}{(1+|z|)^{2}},
$$

it is straightforward to check that

$$
\left|\frac{w^{\prime}}{w}+\eta\right| \leq \tilde{\varepsilon} \lambda_{0} H, \quad\left|\frac{w^{\prime \prime}}{w}-\eta^{2}\right| \leq \tilde{\varepsilon} \lambda_{0} H\left(5+\tilde{\varepsilon} \lambda_{0} H\right)
$$

It follows that there exists $h>0$, depending on the desired terms, such that

$$
\begin{gathered}
\frac{P v}{w} \leq \gamma \eta \varphi_{\eta}-\operatorname{Tr}\left(A D^{2} \varphi_{\eta}\right)+2 \eta e A D \varphi_{\eta}-\eta^{2} e A e \varphi_{\eta}+q \cdot\left(-e \eta \varphi_{\eta}+D \varphi_{\eta}\right)-g(t) \varphi_{\eta} \\
+\tilde{\varepsilon} h\left(\varphi_{\eta}+\left|\dot{\varphi}_{\eta}\right|+\left|D \varphi_{\eta}\right|+\left|\dot{\varphi}_{\eta}\right|+\left|D \dot{\varphi}_{\eta}\right|+\left|\ddot{\varphi}_{\eta}\right|\right) .
\end{gathered}
$$

Whence, recalling that $L_{\eta} \varphi_{\eta}=k(\eta) \varphi_{\eta}$,

$$
\frac{P v}{w} \leq(\gamma \eta-k(\eta)-g(t)) \varphi_{\eta}+\tilde{\varepsilon} h\left(\varphi_{\eta}+\left|\dot{\varphi}_{\eta}\right|+\left|D \varphi_{\eta}\right|+\left|\dot{\varphi}_{\eta}\right|+\left|D \dot{\varphi}_{\eta}\right|+\left|\ddot{\varphi}_{\eta}\right|\right) .
$$

Therefore, applying Theorem 3.3 and noticing that, by Harnack's inequality and periodicity, the $\left(\varphi_{\lambda}\right)_{0<\lambda \leq \lambda_{0}}$ are uniformly bounded away from 0 , we can find a constant $h^{\prime}$ depending on the desired terms such that

$$
\frac{P v}{v} \leq(\gamma \eta-k(\eta)-g(t))+h^{\prime} \tilde{\varepsilon} .
$$

By (3.9), (3.10) there exists a function $\sigma \in W^{1, \infty}\left(\mathbb{R}_{+}\right)$such that

$$
\inf _{\mathbb{R}_{+}}\left(\sigma^{\prime}+g\right) \geq \max _{0 \leq \lambda \leq \lambda_{0}}(\gamma \lambda-k(\lambda))+\varepsilon, \quad\|\sigma\|_{L^{\infty}\left(\mathbb{R}_{+}\right)} \leq 2 T\|g\|_{L^{\infty}\left(\mathbb{R}_{+}\right)} .
$$

Consequently, the function $\underline{v}$ defined by $\underline{v}(x, t):=e^{-\sigma(t)} v(x, t)$ satisfies, for $t>0$,

$$
\frac{P \underline{v}}{\underline{v}}=-\sigma^{\prime}+\frac{P v}{v} \leq \gamma \eta-k(\eta)-\left(\sigma^{\prime}+g\right)(t)+h^{\prime} \tilde{\varepsilon} \leq-\varepsilon+h^{\prime} \tilde{\varepsilon} .
$$

Choosing $\tilde{\varepsilon}=\varepsilon / h^{\prime}$ we eventually infer that $\underline{v}$ is a subsolution of (3.8). We finally renormalize $\underline{v}$ in such a way that it satisfies the bounds stated in the lemma, with $\kappa$ depending on the desired terms.

Next, assume that there exists a generalized transition wave with speed $c$ and profile $\phi$. It satisfies

$$
\partial_{t} \phi-\operatorname{Tr}\left(A_{c}(x, t) D^{2} \phi\right)+q_{c}(x, t) \cdot D \phi=f(t, \phi), \quad x \in \mathbb{R}^{N}, t \in \mathbb{R},
$$

with

$$
A_{c}(x, t)=A\left(x+e \int_{0}^{t} c(s) d s\right), \quad q_{c}(x, t)=q\left(x+e \int_{0}^{t} c(s) d s\right)-c(t) e .
$$

Lemma 3.1 implies that the set $\Lambda_{\phi}$ defined at the beginning of Section 3.2 is nonempty (note that, up to a translation in the direction $e$, the function $\phi$ satisfies

$$
\inf _{\substack{x \in e \leq 1 \\ t \in \mathbb{\mathbb { R }}}} \phi(x, t)>0,
$$


because $\phi(x, t) \rightarrow 1$ as $x \cdot e \rightarrow-\infty$ uniformly in $t \in \mathbb{R})$. The proof of Theorem 1.3 is completed by the following estimate, obtained by using the subsolution $\underline{v}$.

Proposition 3.5. Assume that (1.6)-(1.8) and (1.9) hold and that $\lfloor\mu\rfloor>0$. Let $c$ and $\phi$ be the speed and the profile of a generalized transition wave. Then

$$
\lfloor c\rfloor \geq \min _{0<\lambda \leq \inf \Lambda_{\phi}} \frac{\lfloor\mu\rfloor+k(\lambda)}{\lambda} .
$$

Proof. Let $u$ be a generalized transition wave with speed $c$ and profile $\phi$ and let $\lambda_{0} \in \Lambda_{\phi}$. For $0<\varepsilon<\frac{1}{3}\lfloor\mu\rfloor$ set

$$
\gamma:=\min _{0<\lambda \leq \lambda_{0}} \frac{\lfloor\mu\rfloor-3 \varepsilon+k(\lambda)}{\lambda} .
$$

By the definition of least mean, there exists $T>0$ such that

$$
\forall T^{\prime} \geq T, \quad \frac{1}{T^{\prime}} \inf _{t \in \mathbb{R}} \int_{t}^{t+T^{\prime}} c(s) d s<\lfloor c\rfloor+\varepsilon, \quad \forall t \in \mathbb{R}, \quad \frac{1}{T} \int_{t}^{t+T} \mu(s) d s>\lfloor\mu\rfloor-\varepsilon .
$$

For $n \in \mathbb{N}$, let $t_{n}$ be such that

$$
\frac{1}{n T} \int_{t_{n}}^{t_{n}+n T} c(s) d s<\lfloor c\rfloor+\varepsilon .
$$

Let $v_{n}$ be the subsolution of Lemma 3.4 with $g(t)=\mu\left(t+t_{n}\right)-\varepsilon$ and $A(x), q(x)$ replaced by $A\left(x+e \int_{0}^{t_{n}} c(s) d s\right), q\left(x+e \int_{0}^{t_{n}} c(s) d s\right)$ respectively. Because

$$
\inf _{t>0} \frac{1}{T} \int_{t}^{t+T} g(s) d s \geq\lfloor\mu\rfloor-2 \varepsilon=\max _{0 \leq \lambda \leq \lambda_{0}}(\gamma \lambda-k(\lambda))+\varepsilon,
$$

the associated constant $0<\kappa<1$ is independent of $n$ (notice that $k(\lambda)$ is invariant by translations of the coefficients $A, q$ ). Let $\beta>0$ be such that $\phi(x, t) \geq \beta e^{-\lambda_{0} x \cdot e}$, for $x \cdot e>0, t \in \mathbb{R}$. Because $\phi(x, t) \rightarrow 1$ as $x \cdot e \rightarrow-\infty$, uniformly in $x \perp e$ and $t \in \mathbb{R}$, the Harnack inequality yields

$$
\forall \rho \in \mathbb{R}, \quad \inf _{\substack{x \cdot e<\rho \\ t \in \mathbb{R}}} \phi(x, t)>0 .
$$

It is then possible to decrease $\beta$ in such a way that $\phi(x, t) \geq \beta$, for $x \cdot e \leq 0, t \in \mathbb{R}$. Finally, by the regularity hypothesis on $f$, we can further assume that $f(t, w) \geq(\mu(t)-$ $\varepsilon) w$ for $w \in[0, \beta]$. As a consequence, the functions $\left(\theta_{n}\right)_{n \in \mathbb{N}}$ defined by

$$
\forall x \in \mathbb{R}^{N}, t>t_{n}, \quad \theta_{n}(x, t):=\beta v_{n}\left(x+e \int_{t_{n}}^{t} c(s) d s, t-t_{n}\right),
$$

satisfy $\theta_{n}\left(x, t_{n}\right) \leq \phi\left(x, t_{n}\right)$ and

$$
\partial_{t} \theta_{n}-\operatorname{Tr}\left(A_{c}(x, t) D^{2} \theta_{n}\right)+q_{c}(x, t) \cdot D \theta_{n} \leq(\mu(t)-\varepsilon) \theta_{n} \leq f\left(t, \theta_{n}\right), \quad x \in \mathbb{R}^{N}, t>t_{n} .
$$

The comparison principle then yields

$$
\forall n \in \mathbb{N}, x \in \mathbb{R}^{N}, t>t_{n}, \quad \phi(x, t) \geq \theta_{n}(x, t) .
$$


Hence, letting $R \in \mathbb{R}$ be such that $\phi(x, t)<\beta \kappa$ for $x \cdot e \geq R, t \in \mathbb{R}$, we derive

$$
\forall n \in \mathbb{N}, x \cdot e \geq R, t>t_{n}, \quad \beta \kappa>\theta_{n}(x, t)=\beta v_{n}\left(x+e \int_{t_{n}}^{t} c(s) d s, t-t_{n}\right) .
$$

It follows from the properties of $v_{n}$ that $x \cdot e+\int_{t_{n}}^{t} c(s) d s>\gamma\left(t-t_{n}\right)$, for $n \in \mathbb{N}, x \cdot e \geq R$, $t>t_{n}$. Whence, using (3.12),

$$
\forall n \in \mathbb{N}, \quad \gamma n T<R+\int_{t_{n}}^{t_{n}+n T} c(s) d s \leq R+n T(\lfloor c\rfloor+\varepsilon) .
$$

Letting $n \rightarrow \infty$ we eventually obtain $\gamma \leq\lfloor c\rfloor+\varepsilon$ and then

$$
\lfloor c\rfloor \geq \min _{0<\lambda \leq \lambda_{0}} \frac{\lfloor\mu\rfloor+k(\lambda)}{\lambda},
$$

due to the arbitrariness of $\varepsilon$. Because this is true for all $\lambda_{0} \in \Lambda_{\phi}$ and $\inf \Lambda_{\phi}>0$ by Lemma 3.2, the statement follows.

The non-existence statement in Theorem 1.3 is an immediate consequence of Proposition 3.5.

3.5. The non-periodic case. Finally, we prove the non-existence statement in Theorem 1.5. In the non-periodic case the role of $k(\lambda)$ is played by the generalized principal eigenvalue $\tilde{k}(\lambda)$ defined by (1.17). Theorem 1.5 follows from an estimate on the speed of the generalized transition wave with profile $\phi$ in terms of the bottom of the set $\Lambda_{\phi}^{-}$(defined at the beginning of Section 3.2).

Proposition 3.6. Assume that (1.6)-(1.8) hold and that $\lfloor\mu\rfloor_{-}>0$. Let $c$ and $\phi$ be the speed and the profile of a generalized transition wave and let $\lambda_{0}:=\inf \Lambda_{\phi}^{-}$. Then

$$
\lceil c\rceil_{-} \geq \frac{\lfloor\mu\rfloor_{-}+\tilde{k}\left(\lambda_{0}\right)}{\lambda_{0}} .
$$

Proof. The functions $c$ and $\phi$ satisfy (3.11). Applying Lemma 3.1 with $h(x, t)=$ $\frac{f(t, \phi)}{\phi}$ we see that $\Lambda_{\phi}^{-} \neq \emptyset$. Because (3.4) holds with $g=\mu-\frac{1}{2}\lfloor\mu\rfloor$, by Lemma 3.2 we get $\lambda_{0}:=\inf \Lambda_{\phi}^{-}>0$. We divide the rest of the proof into two parts.

Step 1: the speed c satisfies $\sup _{t<0}\left(\lambda_{0} c(t)-\mu(t)\right) \geq \tilde{k}\left(\lambda_{0}\right)$.

Suppose for the sake of a contradiction that $\sup _{t<0}\left(\lambda_{0} c(t)-\mu(t)\right)<\tilde{k}\left(\lambda_{0}\right)$. We will show that there exist $\delta, \varepsilon>0$ such that

$$
\lambda_{0}<\lambda<\lambda_{0}+\delta \Rightarrow \lambda-\varepsilon \in \Lambda_{\phi}^{-}
$$

which is in contradiction with $\lambda_{0}=\inf \Lambda_{\phi}^{-}$. By the continuity of $\tilde{k}$, given by Corollary A.3, there exist $\delta, \beta>0$ such that

$$
\forall \lambda \in\left[\lambda_{0}, \lambda_{0}+\delta\right], t<0, \quad \lambda c(t) \leq \mu(t)+\tilde{k}(\lambda)-3 \beta .
$$

Take $\lambda \in\left(\lambda_{0}, \lambda_{0}+\delta\right] \subset \Lambda_{\phi}^{-}$. There exists $m_{\lambda}>0$ such that

$$
\forall x \in \mathbb{R}^{N}: x \cdot e>0, \forall t<0, \quad \phi(x, t) \geq m_{\lambda} e^{-\lambda x \cdot e} .
$$


By the definition (1.17) of $\tilde{k}(\lambda)$ there exists a function $\varphi \in W_{l o c}^{2, N}\left(\mathbb{R}^{N}\right)$ satisfying

$$
\|\varphi\|_{L^{\infty}\left(\mathbb{R}^{N}\right)}=1, \quad \inf _{\mathbb{R}^{N}} \varphi>0, \quad L_{\lambda} \varphi \geq(\tilde{k}(\lambda)-\beta) \varphi \quad \text { in } \mathbb{R}^{N} .
$$

Let $m^{\prime} \in\left(0, m_{\lambda}\right]$ be such that

$$
\forall u \in\left(0, m^{\prime}\right], \forall t<0, \quad f(t, u) \geq(\mu(t)-\beta) u, \quad m^{\prime} \leq \inf _{\substack{x \in \mathbb{R}^{N}: x \cdot e=0 \\ t<0}} \phi(x, t) .
$$

We consider a family of functions $\left(\theta_{n}\right)_{n \in \mathbb{N}}$ defined as follows:

$$
\forall x \in \mathbb{R}^{N}, t>-n, \quad \theta_{n}(x, t):=m^{\prime} e^{-\left(\lambda-\varepsilon \chi_{n}(x, t)\right) x \cdot e} \varphi\left(x+e \int_{0}^{t} c(s) d s\right),
$$

with $\varepsilon \in\left(0, \lambda_{0}\right)$ to be chosen later and $\chi_{n}(x, t):=\chi\left(\frac{x \cdot e+2}{t+n+1}\right)$, where $\chi \in C^{2}(\mathbb{R})$ satisfies

$$
\chi=1 \text { in }(-\infty, 1), \quad \chi=0 \text { in }(2,+\infty), \quad \chi^{\prime} \leq 0 \text { in } \mathbb{R} .
$$

We claim that, for $\varepsilon$ small enough, independent of $\lambda$ and $n, \theta_{n}$ is a subsolution of (3.11) for $x \cdot e>0$ and $-n<t<0$. Because $\theta_{n} \leq m^{\prime}$ for $x \cdot e \geq 0$, it is sufficient to show that

$$
\partial_{t} \theta_{n}-\operatorname{Tr}\left(A_{c} D^{2} \theta_{n}\right)+q_{c} \cdot D \theta_{n} \leq(\mu-\beta) \theta_{n}, \quad x \cdot e>0,-n<t<0 .
$$

Setting $\varphi_{c}(x, t):=\varphi\left(x+e \int_{0}^{t} c(s) d s\right)$, explicit computation yields

$$
\begin{aligned}
\partial_{t} \theta_{n}-\operatorname{Tr}\left(A_{c} D^{2} \theta_{n}\right)+q_{c} \cdot D \theta_{n}=m^{\prime} & e^{-\left(\lambda-\varepsilon \chi_{n}\right) x \cdot e}\left(-L_{\lambda} \varphi_{c}+\lambda c \varphi_{c}\right) \\
& -2 \varepsilon m^{\prime} e^{-\left(\lambda-\varepsilon \chi_{n}\right) x \cdot e}\left[\chi_{n} e A_{c} D \varphi_{c}+(x \cdot e) D \chi_{n} A_{c} D \varphi_{c}\right] \\
& +\varepsilon \theta_{n} x \cdot e\left[\partial_{t} \chi_{n}-\operatorname{Tr}\left(A_{c} D^{2} \chi_{n}\right)-\varepsilon(x \cdot e) D \chi_{n} A_{c} D \chi_{n}\right] \\
& -\varepsilon \theta_{n}\left[2(1+x \cdot e) e A_{c} D \chi_{n}+\left(\varepsilon \chi_{n}^{2}-2 \lambda \chi_{n}\right) e A_{c} e\right] \\
& +\varepsilon \theta_{n} q_{c} \cdot\left[(x \cdot e) D \chi_{n}+e \chi_{n}\right] .
\end{aligned}
$$

Using the fact that $\chi_{n}(x, t)=0$ if $x \cdot e>2(t+n)$, one sees that if $x \cdot e>0$ and $t>-n$ then

$$
x \cdot e\left|\partial_{t} \chi_{n}\right| \leq 4\left\|\chi^{\prime}\right\|_{\infty}, \quad(x \cdot e+2)\left|D \chi_{n}\right| \leq 2\left\|\chi^{\prime}\right\|_{\infty}, \quad x \cdot e\left|D_{i j} \chi_{n}\right| \leq 2\left\|\chi^{\prime \prime}\right\|_{\infty} .
$$

Moreover, by Theorem A.1 in the Appendix, we may assume that $\varphi$ satisfies $|D \varphi| \leq \zeta \varphi$, for some $\zeta$ depending on $N, \alpha, \lambda_{0}$, and the $L^{\infty}$ norms of $A$ and $q$. Consequently, there exists $C>0$, depending on the same parameters as well as on $\|\chi\|_{W^{2, \infty}}$, such that for $x \cdot e>0$ and $-n<t<0$,

$$
\begin{aligned}
\partial_{t} \theta_{n}-\operatorname{Tr}\left(A_{c} D^{2} \theta_{n}\right)+\left[q_{\tilde{c}}-c e\right] \cdot D \theta_{n} & \leq m^{\prime} e^{-\left(\lambda-\varepsilon \chi_{n}\right) x \cdot e}\left(-L_{\lambda} \varphi_{c}+\lambda c \varphi_{c}\right)+C(1+\varepsilon+\lambda) \varepsilon \theta_{n} \\
& \leq\left[-\tilde{k}(\lambda)+\beta+\lambda c+C\left(1+\varepsilon+2 \lambda_{0}\right) \varepsilon\right] \theta_{n} \\
& \leq\left[\mu-2 \beta+C\left(1+\varepsilon+2 \lambda_{0}\right) \varepsilon\right] \theta_{n} .
\end{aligned}
$$

Therefore (3.14) holds for $\varepsilon$ small enough, independent of $\lambda$ and $n$. For the boundary and initial conditions, we have that

$$
\forall x \in \mathbb{R}^{N}: x \cdot e=0,-n<t<0, \quad \theta_{n}(x, t) \leq m^{\prime} \leq \phi(x, t),
$$


$\forall x \in \mathbb{R}^{N}: x \cdot e>0, \quad \theta_{n}(x,-n)=m^{\prime} e^{-\lambda x \cdot e} \varphi\left(x+e \int_{0}^{-n} c(s) d s\right) \leq m_{\lambda} e^{-\lambda x \cdot e} \leq \phi(x,-n)$.

Thus, the comparison principle yields

$$
\forall x \in \mathbb{R}^{N}: x \cdot e>0,-n<t<0, \quad \phi(x, t) \geq m^{\prime} e^{-\left(\lambda-\varepsilon \chi_{n}(x, t)\right) x \cdot e} \varphi\left(x+e \int_{0}^{t} c(s) d s\right),
$$

whence, letting $n$ go to infinity, we obtain

$$
\forall x \in \mathbb{R}^{N}: x \cdot e>0, t<0, \quad \phi(x, t) \geq m^{\prime}\left(\min _{\mathbb{R}^{N}} \varphi\right) e^{-(\lambda-\varepsilon) x \cdot e} .
$$

That is, $\lambda-\varepsilon \in \Lambda_{\phi}^{-}$.

Step 2: the speed c satisfies $\lceil c\rceil_{-} \geq \frac{\lfloor\mu\rfloor_{-}+\tilde{k}\left(\lambda_{0}\right)}{\lambda_{0}}$.

Take $\sigma \in W^{1, \infty}(\mathbb{R})$ and consider the function $\tilde{c}:=c-\sigma^{\prime}$. Because $\sigma$ is bounded and

$$
\tilde{\phi}(x, t):=u\left(x+e \int_{0}^{t} \tilde{c}(s) d s, t\right)=\phi(x-e(\sigma(t)-\sigma(0)), t),
$$

it follows from Definition 1.1 that $\tilde{c}$ is another admissible speed for $u$, associated with the profile $\tilde{\phi}$. Furthermore, because by the Harnack inequality the profile of a generalized transition wave satisfies (3.13), we see that $\Lambda_{\tilde{\phi}}^{-}=\Lambda_{\phi}^{-}$. In particular $\inf \Lambda_{\tilde{\phi}}^{-}=\lambda_{0}$. We can then apply Step 1 to the speed $\tilde{c}$ and infer that $\sup _{t<0}\left(\lambda_{0} \tilde{c}(t)-\right.$ $\mu(t)) \geq \tilde{k}\left(\lambda_{0}\right)$. We have shown that

$$
\forall \sigma \in W^{1, \infty}(\mathbb{R}), \quad \inf _{t<0}\left(\frac{\mu(t)+\tilde{k}\left(\lambda_{0}\right)}{\lambda_{0}}-c(t)+\sigma^{\prime}(t)\right) \leq 0 .
$$

Owing to (3.2), we get

$$
0 \geq\left\lfloor\frac{\mu+\tilde{k}\left(\lambda_{0}\right)}{\lambda_{0}}-c\right\rfloor_{-} \geq \frac{\lfloor\mu\rfloor_{-}+\tilde{k}\left(\lambda_{0}\right)}{\lambda_{0}}-\lceil c\rceil_{-} .
$$

REMARK 3.2. The fact that Theorem 1.5 involves the upper mean of $c$ rather than the least mean is only due to the very last inequality in the proof: $\left\lfloor\frac{\mu}{\lambda_{0}}-c\right\rfloor_{-} \geq$ $\frac{\lfloor\mu\rfloor_{-}}{\lambda_{0}}-\lceil c\rceil_{-}$, which is in general far from sharp. Additional information on $c$ could possibly improve the result.

REMARK 3.3. One can slightly improve Theorem 1.5 by replacing $c, \mu$ with any functions $\hat{c}, \hat{\mu}$ obtained as the limits of the translations $c\left(\cdot+t_{n}\right)$ and $\mu\left(\cdot+t_{n}\right)$.

Appendix A. Properties of $\tilde{k}(\lambda)$. In this section, we derive some properties of the generalized principal eigenvalue $\tilde{k}$ associated with general linear elliptic operators of the type

$$
\mathcal{L} \psi=\operatorname{Tr}\left(A(x) D^{2} \psi\right)+q(x) \cdot D \psi+h(x) \psi
$$


The definition of the generalized principal eigenvalue is

$$
\tilde{k}(\mathcal{L}):=\sup \left\{k \in \mathbb{R}: \exists \varphi \in W_{l o c}^{2, N}\left(\mathbb{R}^{N}\right) \cap L^{\infty}\left(\mathbb{R}^{N}\right), \inf _{\mathbb{R}^{N}} \varphi>0, \mathcal{L} \varphi \geq k \varphi \text { in } \mathbb{R}^{N}\right\} .
$$

With this notation, definition $(1.17)$ rewrites as $\tilde{k}(\lambda)=\tilde{k}\left(L_{\lambda}\right)$. The quantity $\tilde{k}(\mathcal{L})$ is a well defined real number due to the following result. The second part of the result is used in the proof of Theorem 1.5.

Theorem A.1. Assume that $A$ satisfies (1.6) and that there exists $\beta>0$ such that

$$
\|A\|_{L^{\infty}\left(\mathbb{R}^{N}\right)},\|q\|_{L^{\infty}\left(\mathbb{R}^{N}\right)},\|h\|_{L^{\infty}\left(\mathbb{R}^{N}\right)} \leq \beta .
$$

Then

$$
\inf _{\mathbb{R}^{N}} h \leq \tilde{k}(\mathcal{L}) \leq \sup _{\mathbb{R}^{N}} h
$$

Moreover, there exists $\zeta>0$, depending on $N$, $\alpha$, and $\beta$, such that the definition (1.17) of $\tilde{k}(\mathcal{L})$ does not change if one further requires

$$
\varphi \in W_{\text {loc }}^{2, p}\left(\mathbb{R}^{N}\right), \forall p<\infty, \quad|D \varphi| \leq \zeta \varphi \text { in } \mathbb{R}^{N} .
$$

Proof. By definition, for $\tilde{k}(\mathcal{L})-1<k<\tilde{k}(\mathcal{L})$, there exists a function $\varphi \in$ $W_{\text {loc }}^{2, N}\left(\mathbb{R}^{N}\right)$ satisfying

$$
\|\varphi\|_{L^{\infty}\left(\mathbb{R}^{N}\right)}=1, \quad \inf _{\mathbb{R}^{N}} \varphi>0, \quad \mathcal{L} \varphi \geq k \varphi \text { in } \mathbb{R}^{N}
$$

Consider the following function:

$$
g(u):= \begin{cases}0, & \text { if } u \leq 1 \\ (u-1)^{2}, & \text { otherwise }\end{cases}
$$

The function $\varphi$ is a subsolution of the equation

$$
(\mathcal{L}-k) \psi=g(\psi) \quad \text { in } \mathbb{R}^{N}
$$

and the constant function $M$ is a supersolution of the same equation, for $M \geq 1$ large enough depending on $\beta$ and $\tilde{k}(\mathcal{L})$. As a consequence, a standard iterative method provides a solution $\varphi \leq \psi \leq M$. In particular, $\mathcal{L} \psi \geq k \psi$ in $\mathbb{R}^{N}$. In order to apply the classical regularity theory, we write the equation for $\psi$ in linear form:

$$
\left(\mathcal{L}-k-\frac{g(\psi)}{\psi}\right) \psi=0 \quad \text { in } \mathbb{R}^{N}
$$

Notice that $0 \leq \frac{g(\psi)}{\sim} \leq M-1$. Thus, $\psi \in W_{\mathrm{loc}}^{2, p}\left(\mathbb{R}^{N}\right), \forall p<\infty$. Due to the arbitrariness of $k \in(\tilde{k}(\mathcal{L})-1, \tilde{k}(\mathcal{L}))$, this shows that requiring this extra condition on $\varphi$ does not affect definition (1.17). By Moser's inequality, we can find a constant $\zeta^{\prime}$, only depending on $N, \alpha, \beta$, and $\tilde{k}(\mathcal{L})$, such that

$$
\forall x \in \mathbb{R}^{N}, \quad\|\psi\|_{C^{1}\left(B_{1}(x)\right)} \leq \zeta^{\prime}\|\psi\|_{L^{\infty}\left(B_{2}(x)\right)} .
$$


Then, using Harnack's inequality, we can find another constant $\zeta$, depending on the same parameters, such that

$$
\forall x \in \mathbb{R}^{N}, \quad\|\psi\|_{C^{1}\left(B_{1}(x)\right)} \leq \zeta \psi(x) .
$$

Therefore, to conclude the proof it is sufficient to show that (A.1) holds. The first inequality simply follows by taking $\varphi \equiv 1$ in the definition. Let $\left(x_{n}\right)_{n \in \mathbb{N}}$ be a maximizing sequence for $\psi$. The functions $\psi\left(\cdot+x_{n}\right)$ converge as $n \rightarrow \infty$ (up to subsequences) locally uniformly in $\mathbb{R}^{N}$ to a function $\tilde{\psi}$ having positive maximum at 0 and satisfying

$$
\operatorname{Tr}\left(\tilde{A}(x) D^{2} \tilde{\psi}\right)+\tilde{q}(x) \cdot D \tilde{\psi}+\tilde{h}(x) \tilde{\psi} \geq k \tilde{\psi} \quad \text { in } \mathbb{R}^{N},
$$

where $\tilde{A}, \tilde{q}, \tilde{h}$ are the local limits of (subsequences of) $A\left(\cdot+x_{n}\right), q\left(\cdot+x_{n}\right), h\left(\cdot+x_{n}\right)$ (in the weak sense in the case of $q$ and $h$ ). Taking the limessinf as $x \rightarrow 0$ in the above differential inequality and using Bony's maximum principle we get $k \leq \sup _{\mathbb{R}^{N}} \tilde{h} \leq$ $\sup _{\mathbb{R}^{N}} h$. Thus, $\tilde{k}(\mathcal{L}) \leq \sup _{\mathbb{R}^{N}} h$.

A first consequence of Theorem A.1 is that if $\mathcal{L}$ is periodic then $\tilde{k}(\mathcal{L})$ coincides with the periodic principal eigenvalue $k(\mathcal{L})$, i.e., the unique $k$ such that $\mathcal{L} \varphi=k \varphi$ in $\mathbb{R}^{N}$ admits a positive, $l$-periodic solution.

Corollary A.2. If $A$ satisfies (1.6) and $A, q, h \in L^{\infty}\left(\mathbb{R}^{N}\right)$ are l-periodic then $\tilde{k}(\mathcal{L})=k(\mathcal{L})$.

Proof. Let $\varphi_{p}$ be the periodic principal eigenfunction of $\mathcal{L}$. For $\varphi \in W_{\text {loc }}^{2, N}\left(\mathbb{R}^{N}\right)$ and $k \in \mathbb{R}$, setting $\hat{\varphi}:=\varphi / \varphi_{p}$ we see that $\mathcal{L} \varphi \geq k \varphi$ if and only if $\hat{\mathcal{L}} \hat{\varphi} \geq k \hat{\varphi}$, with

$$
\hat{\mathcal{L}}=\operatorname{Tr}\left(A(x) D^{2}\right)+\left(q(x)+2 \frac{A(x) D \varphi_{p}}{\varphi_{p}}\right) \cdot D+k(\mathcal{L}) .
$$

As a consequence $\tilde{k}(\mathcal{L})=\tilde{k}(\hat{\mathcal{L}})=k(\mathcal{L})$, where the last equality follows from (A.1).

We now apply Theorem A.1 to derive the continuity of the function $\lambda \mapsto \tilde{k}(\lambda)$ defined by (1.17).

Corollary A.3. Under the assumptions (1.6), (1.7), the function $\lambda \mapsto \tilde{k}(\lambda)$ is continuous on $\mathbb{R}$. Moreover, for given $m>0, \lambda \mapsto \frac{m+\tilde{k}(\lambda)}{\lambda}$ has a minimum on $\mathbb{R}_{+}$.

Proof. Fix $K, \varepsilon>0$. Let $\lambda, \lambda^{\prime} \in[-K, K]$. By Theorem A.1 there exists $\zeta>0$, depending on $N, \alpha, K$, and the $L^{\infty}$ norms of $A$ and $q$, and a function $\varphi \in W_{\operatorname{loc}}^{2, p}\left(\mathbb{R}^{N}\right) \cap$ $L^{\infty}(\mathbb{R}), \forall p<\infty$, such that

$$
\inf _{\mathbb{R}^{N}} \varphi>0, \quad|D \varphi| \leq \zeta \varphi, \quad L_{\lambda} \varphi \geq(\tilde{k}(\lambda)-\varepsilon) \varphi \quad \text { in } \mathbb{R}^{N}
$$

It follows that

$$
\begin{aligned}
L_{\lambda^{\prime}} \varphi & =L_{\lambda} \varphi+\left(\lambda^{\prime}-\lambda\right)\left[-2 e A(x) D \varphi+\left(\lambda^{\prime}+\lambda\right) e A(x) e+q(x) e\right] \varphi \\
& \geq\left[\tilde{k}(\lambda)-\varepsilon-C(\zeta+2 K+1)\left|\lambda^{\prime}-\lambda\right|\right] \varphi,
\end{aligned}
$$

where $C$ depends on $N$ and the $L^{\infty}$ norms of $A$ and $q$. Thus, if $\left|\lambda^{\prime}-\lambda\right|$ is small enough, independently of $\lambda$ and $\lambda^{\prime}, L_{\lambda^{\prime}} \varphi \geq(\tilde{k}(\lambda)-2 \varepsilon) \varphi$ in $\mathbb{R}^{N}$, that is, $\tilde{k}\left(\lambda^{\prime}\right) \geq \tilde{k}(\lambda)-2 \varepsilon$. This proves the continuity of $\tilde{k}$. 
The last statement of the corollary follows from the continuity of $\tilde{k}$ and the fact that, by (A.1),

$$
\frac{m+\tilde{k}(\lambda)}{\lambda} \geq \frac{m}{\lambda}+\inf _{x \in \mathbb{R}^{N}}(\lambda e A(x) e+q(x) e)
$$

which tends to $+\infty$ as $\lambda \rightarrow 0^{+},+\infty$.

Acknowledgments. L. Ro. was partially supported by GNAMPA - INdAM and the Fondazione CaRiPaRo Project "Nonlinear Partial Differential Equations: models, analysis, and control-theoretic problems", while L. Ry. was partially supported by NSF grant DMS-0908507. L. Ry. thanks Università di Padova for its hospitality.

\section{REFERENCES}

[1] D.G. Aronson and H.F. Weinberger, Multidimensional nonlinear diffusion arising in population genetics, Adv. Math., 30(1), 33-76, 1978.

[2] H. Berestycki and F. Hamel, Front propagation in periodic excitable media, Commun. Pure Appl. Math., 55(8), 949-1032, 2002.

[3] H. Berestycki and F. Hamel, Generalized travelling waves for reaction-diffusion equations, in Perspectives in Nonlinear Partial Differential Equations, Contemp. Math., Amer. Math. Soc., Providence, RI, 446, 101-123, 2007.

[4] H. Berestycki and G. Nadin, Spreading speeds for one-dimensional monostable reactiondiffusion equations, J. Math. Phys., 53(11), 115619, 2012.

[5] H. Berestycki, L. Nirenberg, and S.R.S. Varadhan, The principal eigenvalue and maximum principle for second-order elliptic operators in general domains, Commun. Pure Appl. Math., 47(1), 47-92, 1994.

[6] H. Berestycki and L. Rossi, On the principal eigenvalue of elliptic operators in $\mathbb{R}^{N}$ and applications, J. Eur. Math. Soc. (JEMS), 8(2), 195-215, 2006.

[7] J.M. Bony, Principe du maximum dans les espaces de Sobolev, C.R. Acad. Sci. Paris Sér. A-B, 265, A333-A336, 1967.

[8] R.A. Fisher, The wave of advantage of advantageous genes, Ann. Eugenics, 7, 355-369, 1937.

[9] T. Kato, Perturbation Theory for Linear Operators, Springer-Verlag, Berlin, Second Edition, 1976. Grundlehren der Mathematischen Wissenschaften, Band 132.

[10] A.N. Kolmogorov, I.G. Petrovskiŭ, and N.S. Piskunov, Study of the diffution equation with growth of the quantity of matter and its application to a biological problem, Bull. Univ. Etat. Moscow Ser. Internat. Math. Mec. Sect. A, 1, 1-29, 1937.

[11] J.F. Mallordy and J.M. Roquejoffre, A parabolic equation of the KPP type in higher dimensions, SIAM J. Math. Anal., 26(1), 1-20, 1995.

[12] A. Mellet, J.M. Roquejoffre, and Y. Sire, Generalized fronts for one-dimensionnal reactiondiffusion equations, Disc. Cont. Dyn. Sys., 26(1), 303-312, 2009.

[13] G. Nadin, Traveling fronts in space-time periodic media, J. Math. Pures Appl., 92, 232-262, 2009.

[14] G. Nadin, Critical travelling waves for general heterogeneous one-dimensional reactiondiffusion equations, preprint.

[15] G. Nadin and L. Rossi, Propagation phenomena for time heterogeneous KPP reaction-diffusion equations, J. Math. Pures Appl., (9), 98(6), 633-653, 2012.

[16] J. Nolen, J.M. Roquejoffre, L. Ryzhik, and A. Zlatoš, Existence and non-existence of FisherKPP transition fronts, Arch. Ration. Mech. Anal., 203(1), 217-246, 2012.

[17] J. Nolen, M. Rudd, and J. Xin, Existence of KPP fronts in spatially-temporally periodic advection and variational principle for propagation speeds, Dyn. PDE, 2(1), 1-24, 2005.

[18] J. Nolen and L. Ryzhik, Traveling waves in a one-dimensional heterogeneous medium, Ann. Inst. H. Poincaré Anal. Non Linéaire, 26(3), 1021-1047, 2009.

[19] M. Reed and B. Simon, Methods of Modern Mathematical Physics. IV. Analysis of Operators, Academic Press [Harcourt Brace Jovanovich Publishers], New York, 1978.

[20] W. Shen, Traveling waves in diffusive random media, J. Dyn. Diff. Eqs., 16(4), 1011-1060, 2004.

[21] W. Shen, Existence, uniqueness, and stability of generalized traveling waves in time dependent monostable equations, J. Dyn. Diff. Eqs., 23(1), 1-44, 2011. 


\section{TRANSITION WAVES FOR TIME-DEPENDENT MONOSTABLE EQUATIONS}

[22] W. Shen, Existence of generalized traveling waves in time recurrent and space periodic monostable equations, J. Appl. Anal. Comp., 1(1), 69-93, 2011.

[23] N. Shigesada, K. Kawasaki, and E. Teramoto, Traveling periodic waves in heterogeneous environments, Theor. Population Biol., 30, 143-160, 1986.

[24] J. Xin, Existence of planar flame fronts in convective-diffusive periodic media, Arch. Ration. Mech. Anal., 121, 205-233, 1992. 